\title{
Nonsurgical Retreatment Using Regenerative Endodontic Protocols: A Case Report
}

\author{
Tugba Turk ${ }^{1}$, Andrea Cicconetti ${ }^{2}$, Dario Di Nardo ${ }^{3}$, Miccoli Gabriele ${ }^{4}$, Simone Coppola ${ }^{5}$, Shilpa Bhandi ${ }^{6}$, Marco Seracchiani ${ }^{7}$, \\ Luca Testarelli ${ }^{8}$, Gianluca Gambarini ${ }^{9}$
}

\begin{abstract}
Aim and objective: The aim of this case report was to describe regenerative endodontic procedures (REPs) of the previously treated mature teeth with long-term results.

Background: Regenerative endodontic procedures are aimed to treat apical periodontitis and regenerate the pulp-dentin complex in necrotic teeth. However, there is no consensus in using REPs in the previously treated mature teeth. The aim of this case report was to describe REPs of the previously treated mature teeth with long-term results.

Case description: A 25-year-old woman presented pain on chewing on 15 and swelling (WHO numbering system). The tooth had been endodontically treated and restored 4 years ago. After removing the old root canal filing, the roots were irrigated with $2.5 \%$ sodium hypochlorite and $17 \%$ EDTA using sonic activation. Calcium hydroxide $(\mathrm{CH})$ was used as medicament for 3 weeks. At the second visit, $\mathrm{CH}$ was removed, and canals were irrigated as like as the first visit. Apical bleeding was induced, and concentrated growth factors (CGFs) were placed inside the root canal. The tooth has been followed up to 3 years.

Conclusion: The tooth was functional and asymptomatic at the recall visits. Since the first-year follow-up, the tooth responded to the electric pulp test and the thermal test. Radiological examinations revealed healing of apical lesion and hard tissue deposition.

Clinical significance: Regenerative endodontic procedures can offer an advantage over traditional endodontic procedures in terms of tertiary healing, with a predictable, user-friendly procedure also for retreatment cases.

Keywords: Concentrated growth factor, Endodontic retreatment, Mature tooth, Regenerative endodontics, Revitalization.

The Journal of Contemporary Dental Practice (2020): 10.5005/jp-journals-10024-2909
\end{abstract}

\section{BACKGROUND}

Having persistent or renewed discomfort with an endodontically treated tooth is usually a sign of failure., ${ }^{1,2}$ Pain on percussion is the most typical sign of inflammation of the tissues that surround the tooth's root, and it is commonly related to a situation where infection has reestablished itself inside the tooth and is now affecting ligament and bone. ${ }^{3,4}$ An eventual response to cold or heat in an endodontically treated tooth is usually related to poor elimination of the original pulp tissue (i.e., missed canals); in such cases, proper shaping and cleaning procedures should be the main goal of the retreatment. ${ }^{4}$ Pain on chewing could be also related to overfilling, causing an unnecessary mechanical and chemical irritation, which may hinder repair of the periapical tissue and thus diminishes the probability of a successful prognosis. ${ }^{1,3}$ In such cases, elimination of the extruded material is preferable but is not mandatory, because failure may be more common due to other factors, i.e., a nonhermetic seal. ${ }^{5,6}$

Nonsurgical endodontic retreatment has become a wellestablished therapy, which requires proper diagnosis and procedures to achieve success. ${ }^{7}$ Retreated teeth can function well for years, even for a lifetime; in successful cases, patients are asymptomatic, with no thermal sensitivity. Advances in technology are constantly changing the way the root canal retreatment is performed, aiming at improving quality and outcome; therefore, endodontists may nowadays use new techniques that were not available when patients had their first procedure. ${ }^{8,9}$ In the present case report, the goal was to use innovative regenerative endodontic protocols (REPs) in a retreatment case, aiming not only to achieve an asymptomatic tooth and healing of the periapical lesion but
${ }^{1}$ Department of Endodontics, EGE University, Izmir, Turkey

${ }^{2-5,7-9}$ Department of Oral and Maxillo-facial Sciences, Sapienza University of Rome, Rome, Italy

${ }^{6}$ Department of Restorative Dental Sciences, Division of Operative Dentistry, College of Dentistry, Jazan University, Jazan, Kingdom of Saudi Arabia

Corresponding Author: Marco Seracchiani, Department of Oral and Maxillo-facial Sciences, Sapienza University of Rome, Rome, Italy, Phone: +39334 379 5174, e-mail: marco.seracchiani@uniroma1.it

How to cite this article: Turk T, Cicconetti A, Di Nardo D, et al. Nonsurgical Retreatment Using Regenerative Endodontic Protocols: A Case Report. J Contemp Dent Pract 2020;21(11):1275-1278.

Source of support: Nil

Conflict of interest: None

also to restore tooth vitality (even if partially). The goal of such an innovative cell-free approach was to allow patient to regain thermal sensitivity in a tooth where previous pulp tissue had been eliminated and substituted with an artificial (gutta-percha and sealer) root canal obturation.

\section{Case Description}

A 25-year-old woman was referred due to pain on chewing on 15 and swelling (WHO numbering system) to the Department of Endodontics of EGE University, Izmir, Turkey. The tooth had been endodontically treated and restored 4 years before. Tooth was sensitive to the percussion test and gave negative response to both 
the thermal and the electrical vitality tests. A periapical radiograph was taken (Fig. 1A), showing a root canal treatment with some overextension of the gutta-percha cones and a periapical lesion related to 1.5. Patient agreed on performing a REP instead of a traditional root canal retreatment after a detailed description of the procedure and the possible advantages and disadvantages, by signing a written informed consent. After the removal of the coronal restoration and the creation of a new access cavity (Fig. 1B), the removal of overextended gutta-percha cone was performed. After that, the working length was determined using an electronic apex locator (Propex II; Dentsply Sirona, Ballaigues, Switzerland). Canal preparation was performed using $\mathrm{Ni}-\mathrm{Ti}$ rotary instruments with $2.5 \%$ sodium hypochlorite irrigation (Merck, Darmstadt, Germany). Special care was given in trying to touch all canal walls and root canal was enlarged up to a \#30.06 final shape. After completing shaping procedure, a final enhanced irrigation procedure was performed alternating the use of $2.5 \%$ sodium hypochlorite and 17\% EDTA (Merck, Darmstadt, Germany) with sonic activation. A calcium hydroxide (CH) (Merck, Darmstadt, Germany) medication was placed, and the patient was given a second appointment 3 weeks later.

At the second visit, the patient was asymptomatic, and a decision was made to complete a cell-free revascularization treatment. Under rubber dam isolation, the temporary restorative material was removed, and the root canal was irrigated with $10 \mathrm{~mL} 2.5 \% \mathrm{NaOCl}$ and $10 \mathrm{~mL} 17 \%$ EDTA with sonic activation. Then, canal was rinsed with saline solution and then dried. Consistent apical bleeding was induced by size 30 manual stainless-steel K-file (Mani, Inc., Tochigi Ken, Japan) and allowed blood to fill endodontic space for 15 minutes and form a blood clot. Then, a collagen sponge was used to slightly dry and compact the blood clot prior to the placement of EndoSequence BC RRM putty (Brasseler USA, Savannah, Georgia, USA) $\sim 2 \mathrm{~mm}$ coronally; finally, the coronal part was sealed (Fig. 1C) with glass ionomer (GC Fuji IX Extra; GC Co., Tokyo, Japan). After 1 month, glass ionomer cement (GIC) was removed, and the cavity was restored with composite resin (Clearfil Majesty Esthetic; Kuraray Medical, Okayama, Japan). Follow-up visits were scheduled every 3 months during the first year and every 6 months for 3 years. At recall appointments, clinical and radiological examinations were performed. In order to acquire more information about apical healing at the 3-year control, a cone beam computed tomography (CBCT) (Kodak 9000 3D; Practice Works, Inc., Atlanta, Georgia, USA) image of the tooth was obtained using standard settings (10.8-second exposure time, $70 \mathrm{kV}$, and $10 \mathrm{~mA}$ ).

During the follow-up evaluations, tooth was functional and asymptomatic; there was no pain on percussion, palpation, and biting. At 1-year follow-up, radiographic examination revealed remarkable healing of apical lesion and hard tissue deposition (Figs 2A and B). The tooth responded to the electric pulp test (Pulptester, Parkell Inc., Edgewood, New York, USA) and the thermal test.

After 36-month follow-up, the tooth continued to be asymptomatic and functional and did not reveal any percussion, palpation, or biting sensitivity. Furthermore, the tooth continued to respond to the thermal and the electric pulp testing. Radiographic examination revealed complete healing of apical lesion and normal periodontal ligament surrounding all aspects of the root (Figs $2 \mathrm{C}$ and 3). Axial, sagittal, and coronal CBCT cross-sections (76 $\mu \mathrm{m}$ thickness) confirmed the apical healing and the apical closure. Both periapical radiographs and $C B C T$ scans revealed that there was no pathological calcification at root canal space.

\section{Discussion}

Regenerative endodontic protocols have been initially proposed for the treatment of vital immature and permanent teeth, then extended for the treatment of immature teeth with necrotic pulp, ${ }^{10}$ and in the last years for the treatment of permanent necrotic teeth. ${ }^{9}$ Even if there is no standard protocol of REPs, the basic approach for necrotic teeth is the first to debride and disinfect properly the root canal system, promote a significant accumulation of undifferentiated stem cells into the canal space by an evokedbleeding second step (usually by over-instrumenting the canal with a manual file) and make a coronal plug with a sealer that can set even in humid/wet environment and creates an hermetic coronal seal, before restoring the tooth. ${ }^{11-13} \mathrm{He}$ et al. demonstrated with several cases the possibility of regenerative endodontic for adult patients. ${ }^{14}$

Based on these premises, authors thought that if these protocols worked in the treatment of permanent necrotic teeth, they could also work in the retreatment cases, because the main difference between the two treatments is only related to the need to remove the preexisting canal obturation and to perform the proper shaping and cleaning, independently from any possible previous iatrogenic error. Moreover, the outcome of a retreatment case was
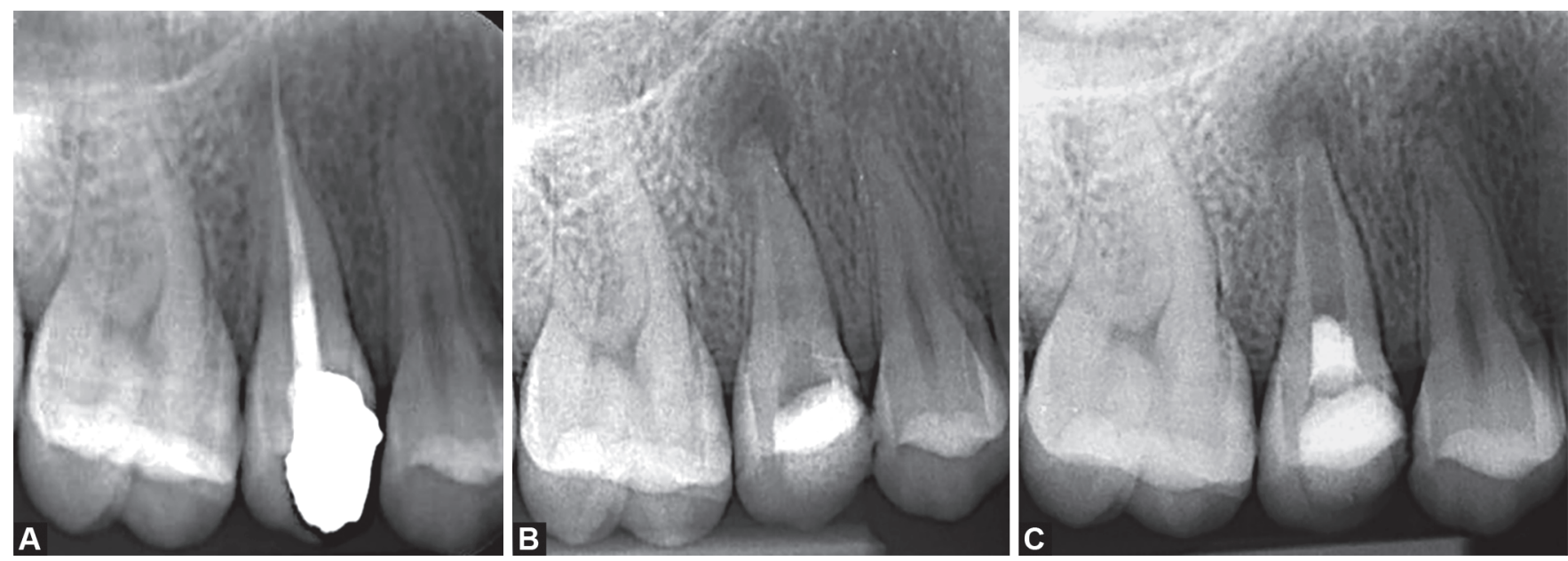

Figs 1A to C: (A) Initial radiograph with previous endodontic treatment and lesion w.r.t. 15; (B) Radiograph after removal of the preexisting obturation, allowing a better visualization of the apical lesion of 15; (C) Final postoperative after regenerative procedure of 15 

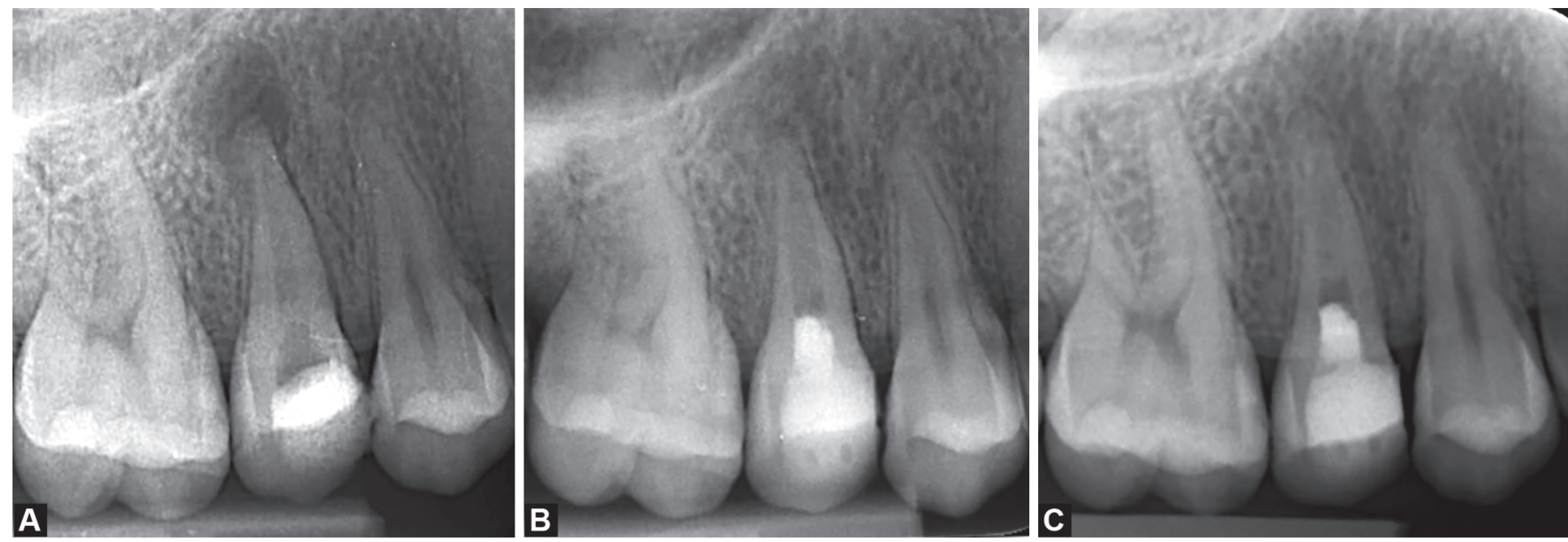

Figs 2A to C: (A) Radiograph following gutta-percha removal of non-vital 15; (B) Postoperative radiograph at 1-year interval presenting vital tooth w.r.t. 15; (C) Three-year interval presenting with primary, secondary, and tertiary healing of 15
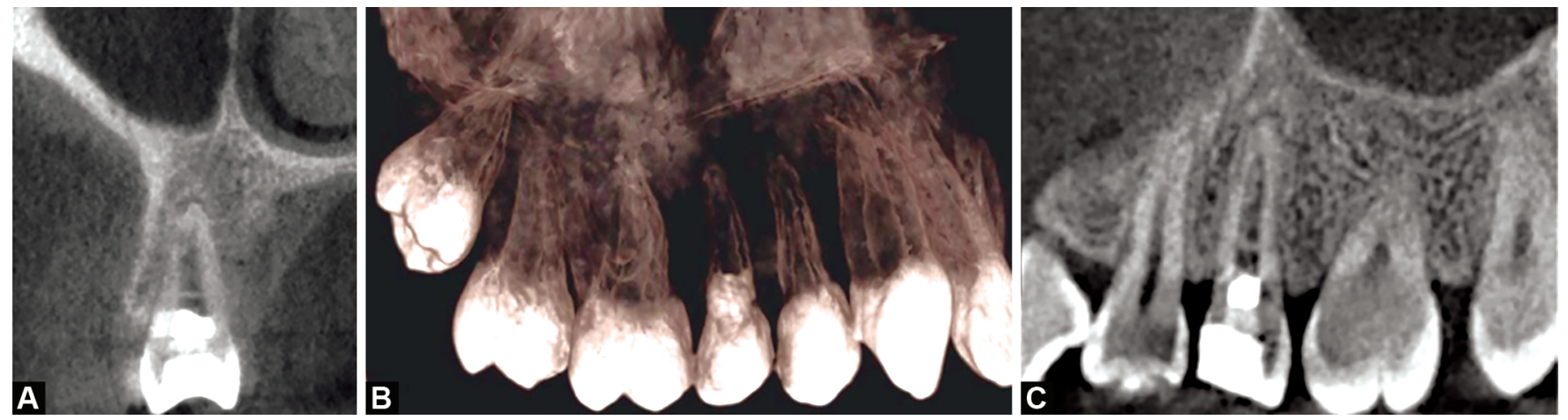

Figs 3 A to C: Cone-beam computed tomography: (A) Image in coronal view; (B) Rendering image; (C) Image in sagittal view of 15, after 3 years, showing the tridimensional primary and secondary healing

thought to be similar to other REPs, provided that the main goals of the endodontic treatment (proper canal debridement, disinfection, and hermetic seal) were respected.

The clinical/radiographic success of REPs has been defined by American Association of Endodontists (AAE), which indicated three main goals (AEE Clinical Consideration). The primary goal (essential) is the elimination of symptoms and the evidence of bony healing. The secondary goal (desirable) is the achievement of an increased root wall thickness and/or increased root length. The tertiary goal is a positive response to vitality testing. ${ }^{15}$ The primary goal is an objective for all endodontic treatments, while the secondary one happens only in immature teeth. However, biological apical closure (assessed by radiographic images) could also be added as a possible secondary goal in permanent teeth. The tertiary (desirable) goal should allow the patient to regain a more natural sensitivity of the tooth and may indicate the development of a tissue more similar to a vital pulp. ${ }^{11}$ This possibility of regaining a positive response to pulp sensitivity testing has been previously reported in some cases. ${ }^{9}$

In the present case report, all the three desirable goals were achieved: the retreated tooth became asymptomatic, showed evidence of bony healing and apical closure, and after some months regained a positive response to the thermal and the electrical pulp sensitivity testing. The fact that a devitalized tooth regained sensitivity and the growth of a sort of new vital tissue inside the endodontic space by a REP offered advantages compared to traditional endodontic retreatment procedures. The patient described the feeling of a more natural tooth, and in this cell-free approach there were no perceived differences between the new procedure and the traditional ones. Placement of the bioceramic coronal plug was a relatively easy and predictable procedure to perform, due to the high consistency and fast set of the putty-like bioceramic material, which has a slight expansion after setting, which contributes to a better hermetic seal; ${ }^{16}$ moreover, the bioceramic obturation has less chance to discolor the tooth and, if necessary, retreatment can be very simple. The use of a cell-free approach was selected because it is more simple, quick, and easy to perform than a cell-based approach, even if the second one is more proper considered to produce a real regeneration. Besides that, impossibility of tooth isolation and extensive loss of tooth structure that require post-support are regarded as contraindications for REPs. ${ }^{15}$

\section{Conclusion}

Since in any endodontic treatment, proper shaping and cleaning procedures are essential for success, special care is only needed to promote bleeding correctly and place a hermetic coronal plug with a material that sets in humid/wet environment. Hence, we may conclude that REPs can offer an advantage over traditional endodontic procedures in terms of tertiary healing, with a simple user-friendly procedure also for the retreatment cases. 


\section{References}

1. Hoen MM, Pink FE. Contemporary endodontic retreatments: an analysis based on clinical treatment findings. J Endod 2002;28(12):834836. DOI: 10.1097/00004770-200212000-00010.

2. Friedman S. Considerations and concepts of case selection in the management of post-treatment endodontic disease (treatment failure). Endod Topics 2002;1(1):54-78. DOI: 10.1034/j.16011546.2002.10105.x.

3. Nair PNR, Sjögren U, Krey G, et al. Intraradicular bacteria and fungi in root-filled, asymptomatic human teeth with therapy-resistant periapical lesions: a long-term light and electron microscopic follow-up study. J Endod 1990;16(12):580-595. DOI: 10.1016/S00992399(07)80201-9.

4. Siqueira J. Aetiology of root canal treatment failure: why well-treated teeth can fail. Int Endod J 2001;34(1):1-10. DOI: 10.1046/j.13652591.2001.00396.x.

5. Ray HA, Trope M. Periapical status of endodontically treated teeth in relation to technical quality of the root filling and the coronal restoration. Int Endod J 1995;28(1):12-18. DOI: 10.1111/j.13652591.1995.tb00150.x.

6. Donfrancesco O, Seracchiani M, Morese A, et al. Analysis of stability in time of marginal adaptation of endosequence root repair material on biological samples. Dent Hypotheses 2020;11:11-15.

7. Song M, Kim HC, Lee W, et al. Analysis of the cause of failure in nonsurgical endodontic treatment by microscopic inspection during endodontic microsurgery. J Endod 2011;37(11):1516-1519. DOI: 10.1016/j.joen.2011.06.032.
8. He L, Kim SG, Gong Q, et al. Regenerative endodontics for adult patients. J Endod 2017;43(9S):57-64. DOI: 10.1016/j.joen.2017.06.012.

9. Arslan $\mathrm{H}$, Ahmed HMA, Şahin $\mathrm{Y}$, et al. Regenerative endodontic procedures in necrotic mature teeth with periapical radiolucencies: a preliminary randomized clinical study. J Endod 2019;45(7):863-872. DOI: 10.1016/j.joen.2019.04.005.

10. Diogenes A, Ruparel NB, Shiloah Y, et al. Regenerative endodontics: a way forward. J Am Dent Assoc 2016;147(5):372-380. DOI: 10.1016/j. adaj.2016.01.009.

11. Diogenes A, Henry MA, Teixeira FB, et al. An update on clinical regenerative endodontics. Endod Topics 2013;28(1):2-23. DOI: 10.1111/etp.12040.

12. Hargreaves KM, Diogenes A, Teixeira FB. Treatment options: biological basis of regenerative endodontic procedures. J Endod 2013;39(3 Suppl):30-43. DOI: 10.1016/j.joen.2012.11.025.

13. Chrepa V, Henry MA, Daniel BJ, et al. Delivery of apical mesenchymal stem cells into root canals of mature teeth. J Dent Res 2015;94(12):16531659. DOI: $10.1177 / 0022034515596527$.

14. Galler KM, Krastl G, Simon S, et al. European society of endodontology position statement: revitalization procedures. Int Endod J 2006;49(8):717-723. DOI: 10.1111/iej.12629.

15. Geisler TM. Clinical considerations for regenerative endodontic procedures. Dent Clin North Am 2012;56(3):603-626. DOI: 10.1016/j. cden.2012.05.010.

16. Lagisetti AK, Hegde P, Hegde MN. Evaluation of bioceramics and zirconia-reinforced glass ionomer cement in repair of furcation perforations: an in vitro study. J Conserv Dent 2018;21(2):184-189. DOI: 10.4103/JCD.JCD_397_16. 